\title{
Pedicle transfer in oral cavity reconstruction
}

\author{
Prabha S. Yadav, Quazi G. Ahmad, Vinay K. Shankhdhar, G. I. Nambi \\ Plastic \& Reconstructive Services, Department of Surgical Oncology, TATA Memorial Hospital, Parel, Mumbai, India
}

Address for correspondence: Dr. G. I. Nambi, Plastic \& Reconstructive Services, Department of Surgical Oncology, TATA Memorial Hospital, Parel, Mumbai - 400 012, India. E-mail: nambi75@rediffmail.com

\section{ABSTRACT}

In head and neck reconstructions when a free flap is used intra orally to provide the lining its vascular pedicle has to be transferred to the neck for anastomosis. This has to be performed in such a way that the pedicle does not get kinked or twisted. The pedicle is enrolled in a split open glove from its point of entry into the flap till its proximal most part. In order to prevent twisting of the vessels and to maintain orientation, the glove is wrapped in such a way that the imprint on the glove is on the visualized surface. The glove wrapped pedicle is passed from inside the oral cavity while an artery clamp passed from the neck wound through the submandibular or subcutaneous tunnel holds the tip of the glove component and guides it safely to the neck without exerting any traction on the flap or the pedicle vessels.

\section{KEY WORDS}

Pedicle transfer; head and neck reconstructions; pedicle tunneling

\section{INTRODUCTION}

econstruction of the intra oral and maxillary defects involves several challenging steps and one among them being the transfer of the vascular pedicle to the neck, the site where the anastomosis is usually performed. Different methods are adopted in various centres across the world for transferring the pedicle vessels to the neck and we would like to share our technique using surgical glove.

After harvesting the free flap, the pedicle vessels are wrapped around in a sterile surgical glove component [Figure 1]. This glove component is made by separating the wrist part of the glove and splitting it open and then trimming it so that the length of the glove component is at least one and a half times longer than that of the pedicle vessels and the width of the glove component is large enough to wrap around [Figure 2] the pedicle vessels. Before wrapping, the pedicle vessels and the inner surface of the glove component are irrigated with saline so that the glove does not slip out while transferring the flap. In order to prevent the twisting of the pedicle and to maintain the orientation of the vessels, an ink mark is made on one side of the glove or the glove can be wrapped in such a way that the imprint on the glove is on one side [Figure 2]. The flap with the glove wrapped pedicle is passed from inside the oral cavity while an artery clamp passed from the neck wound through the submandibular or subcutaneous tunnel holds the tip of the glove component and guides it safely to the neck without exerting any traction on the flap or the pedicle vessels [Figure 3]. When sufficient length of the glove is in the neck, the flap is placed intra orally and the glove component is unwrapped so as to place the flap vessels in the neck [Figure 4]. From this point, both the neck and flap vessels are adjusted accordingly to facilitate the micro anastomosis. The literature search revealed there is only one technique described using the endo tracheal tube ${ }^{[1]}$ for the pedicle transfer, however, this technique using the 


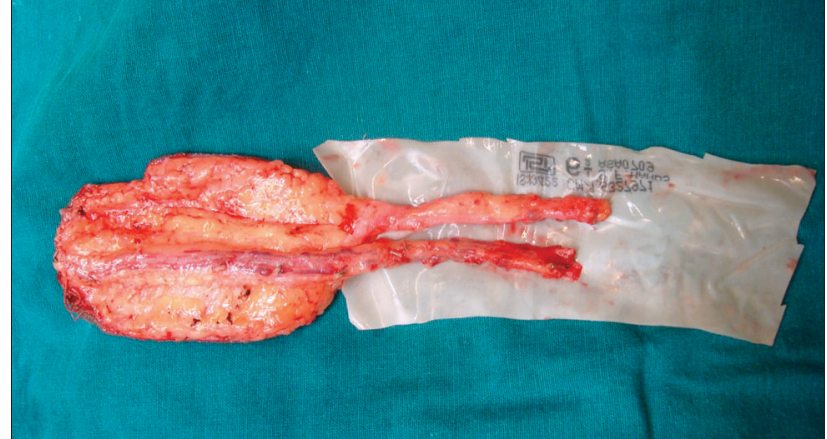

Figure 1: The radial artery forearm flap with its pedicle over the split open wrist part of the surgical gloves. The glove component is at least half the time longer than the pedicle length and is irrigated with saline before the vessels being placed

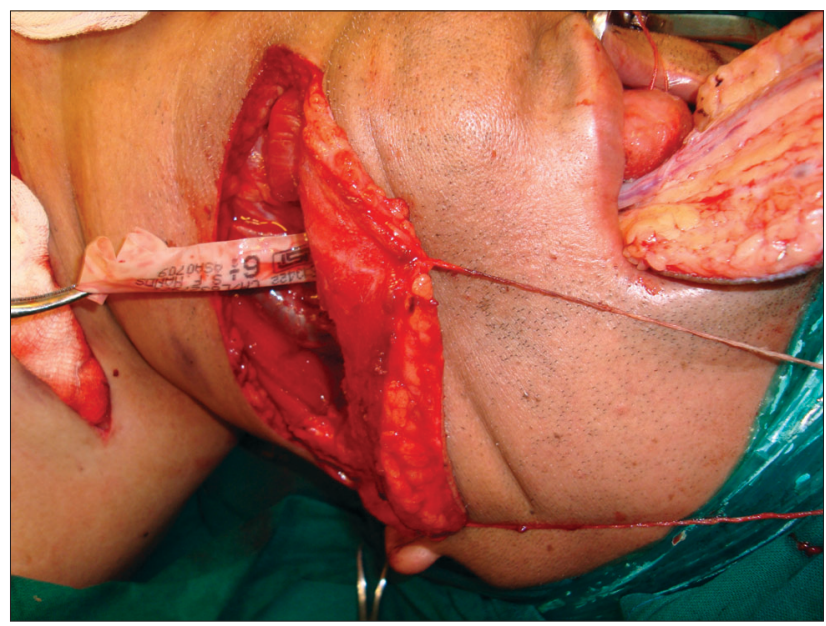

Figure 3: The pedicle is being transferred to the neck with the proximal end being supported in an artery clamp

glove, though commonly followed, is surprisingly not yet reported and is equally safe and effective in transferring nerve grafts and vein grafts beneath the skin bridges wherever required.

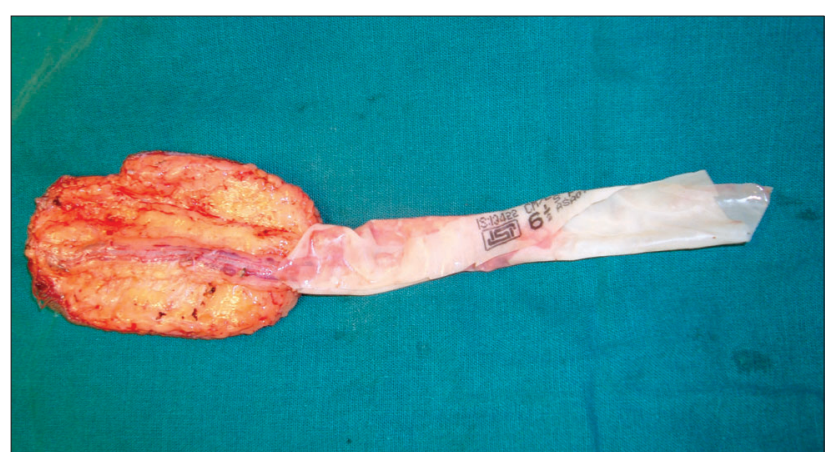

Figure 2: The pedicle is enrolled by the glove from its point of entry into the flap till its proximal most part. In order to prevent twisting of the vessels and to maintain orientation, the glove is wrapped in such a way that the imprint on the glove is on the visualized surface

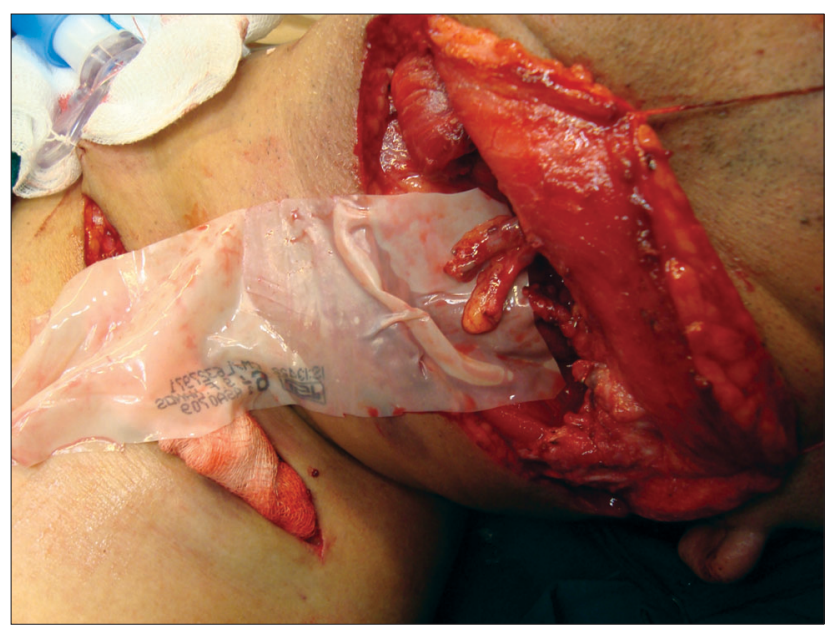

Figure 4: The pedicle in the neck after unwrapping the glove component

\section{REFERENCE}

1. Moschella F, D'Arpa S, Di Lorenzo S, Cordova A. Safe pedicle tunneling in maxillary reconstruction. J Plast Reconstr Aesthet Surg 2010;63:e289. 